\title{
Pathogenic Fitness of Oosporic Progeny Isolates of Phytophthora infestans on Late-Blight-Resistant Tomato Lines
}

\author{
Shiri Klarfeld, Avia (Evgenia) Rubin, and Yigal Cohen, The Mina \& Everard Goodman Faculty of Life Sciences, \\ Bar-Ilan University, Ramat-Gan, Israel
}

\begin{abstract}
Klarfeld, S., Rubin, A. E., and Cohen, Y. 2009. Pathogenic fitness of oosporic progeny isolates of Phytophthora infestans on late-blight-resistant tomato lines. Plant Dis. 93:947-953.
\end{abstract}

Four A1 field isolates and one A2 field isolate of Phytophthora infestans were crossed to produce oospores in tomato leaves. The oospores were extracted and mixed with perlite and water, and healthy tomato leaves were used as bait for oospore-progeny infection. Twenty-nine lesions were obtained from the four crosses and 283 single-sporangium isolates were recovered and tested on four tomato differential lines carrying different major genes $(P h-O, P h-1, P h-2$, and $3707)$ for late blight resistance. The pathogenic fitness (number of sporangia per unit leaf area) of parental and progeny isolates was strongly dependent on the host genotype; it decreased in the order $P h-O>P h-1>P h-2>3707$. The A2 parent had a higher pathogenic fitness than the A1 parents on $P h-O$ and $P h-1$ but similar, lower fitness on $P h-2$. Different levels of pathogenic fitness were observed across all isolates on $P h-0$, although $P h-O$ lacks resistance genes. Pathogenic fitness on one tomato genotype was not related to the pathogenic fitness on the other tomato genotypes. Some isolates exhibited reduced pathogenic fitness relative to the respective A1 parent, whereas others demonstrated a higher pathogenic fitness compared with the A2 parent. The tomato genotype Solanum pimpinellifolium L3707/5 was resistant to all five parental isolates of $P$. infestans. However, 37 of the 283 progeny isolates from 11 different lesions had compatible reactions with this line, producing up to $31 \times 10^{3}$ sporangia/ $\mathrm{cm}^{2}$. Overall, reduced fitness was more frequent among the progeny isolates than increased fitness.

Late blight caused by Phytophthora infestans (Mont.) de Bary (Oomycota, Stramenopiles) is a major disease of potato and tomato worldwide. Mycelium-colonizing seed tubers may initiate the disease in the field whereas sporangia serve as the major vehicle driving epidemics during the season. Oospores, the sexual spores of $P$. infestans, form when $\mathrm{A} 1$ and $\mathrm{A} 2$ isolates mate in infected host tissue, and may be produced in leaves, stems, stolons, tubers, fruit, and seed, as well as in agar cultures $(2,7,8,10,15,19,21,26,27,29,33,35,37)$.

For some regions, oospores play a central role in the epidemiology of late blight in potato $(10,11,19,21,33,37)$ and tomato $(6,14,26,28,29)$. In potato crops growing under field conditions in Israel, oospores were produced in bottom leaves touching the soil but rarely in upper leaves (7). Overhead irrigation enhanced the production of oospores in the upper leaves (7). Oospores were reported to survive in the field as demonstrated by their ability to initiate the late blight disease in situ or in detached leaves in the laboratory $(10,21$,

Corresponding author: Y. Cohen

E-mail: ycohen@mail.biu.ac.il

Accepted for publication 28 May 2009.

doi:10.1094/PDIS-93-9-0947

(C) 2009 The American Phytopathological Society
32,33). In Sweden (32), epidemics were initiated from soilborne oospores. In Central Mexico, where oospores were reported in the 1950s $(13,25)$, soilborne oospores were demonstrated to survive winter fallow and infect potato plants in the field (11). Seedborne oospores can induce late blight infection in tomato seedlings (27). Due to their recombinant nature, oospores may produce new genotypes of the pathogen with altered characteristics, including sensitivity to fungicides, virulence race structure, and aggressiveness, thus changing the epidemiology of the disease $(2,6$, $11,14,15,19,21,30,32,33,37,38)$.

Oospores produced in vivo differed in genotypic properties from oospores produced in agar cultures by the same parents (36). Abundant oospores of $P$. infestans were produced in detached leaves of potato and tomato upon inoculation with mixed $\mathrm{A} 1$ and A2 sporangia (8). In a recent study (29), we reported on an improved method of producing late blight lesions on tomato leaves by floating them as bait on oosporeperlite cultures. This method was used to study the inheritance of resistance to metalaxyl and mating type in the progeny isolates. We demonstrated that, whereas the parent isolates were either sensitive or resistant to metalaxyl, most progeny isolates exhibited intermediate resistance to metalaxyl and belonged to either the A1 or the A2 mating type, strongly suggesting their oosporic origin. The data also con- firmed a large variation in quantitative (compatibility) and qualitative (virulence race structure) traits among the progeny isolates, indicating that some are more compatible or virulent to tomato or potato than their parents.

In Israel, tomato cultivars carrying late blight resistance $(R)$ genes $P h-1$ (e.g., New Yorker) or $P h-2$ (e.g., Pieraline, Macline, and Piline) provide inadequate resistance against the local population of $P$. infestans (6). Farmers rely on frequent fungicide applications for disease control. However, resistance of the pathogen to fungicides (e.g., metalaxyl) often hampers disease control success (15).

A new gene for late blight resistance, $P h-3$, was reported in accession L3708 of Solanum pimpinellifolium (1). Whereas $P h-1$ and $P h-2$ were mapped to chromosomes 7 and 10, respectively (24), $P h-3$ was mapped to chromosome 9 (5). All three genes condition race-specific resistance: Mendelian segregation studies suggested that $P h-1$ is a dominant allele effective against race T0, $P h-2$ is a partially dominant allele highly effective against race $\mathrm{T} 0$ and partially effective against race $\mathrm{T} 1$, and $P h-3$ is a partially dominant allele effective against isolate $\mathrm{Pi}-16$ from Taiwan that overcomes $P h-1$ and $P h-2$ (5). However, later observations made by these authors revealed that L3708 became infected in Taiwan and Nepal; 4 of 59 Taiwanese field isolates of $P$. infestans were virulent on L3708 (5). Chen et al (4) reported that new isolates, aggressive on both potato and tomato, including L3708, evolved in Taiwan during 2004 and 2005.

Kim and Mutschler (18) showed that, whereas tomato lines derived from L3708 were resistant to five isolates of $P$. infestans, the F1 hybrids with a susceptible parent were resistant against one isolate, partially resistant against three other isolates, and susceptible to the fifth isolate, identified as US17. The authors suggested that resistance against US17 requires additional genes besides $P h-3$. Studies conducted in North Carolina by R. Gardner (personal communication) showed that the local population of $P$. infestans has overcome all three $R$ genes against late blight under field conditions.

L3707 is another accession of $S$. pimpinellifolium that is resistant to late blight (1). Data collected in Israel (Y. Cohen, unpublished) confirmed that L3707 was resistant (had low area under the dis- 
ease progress curve values) under late blight epiphytotic conditions in nature. Recent data (16) show that L3707/5 ( $S$. pimpinellifolium L3707/5 = 3707) exhibits resistance against multiple field isolates of $P$. infestans and, therefore, is considered to carry race-nonspecific resistance against late blight. Inheritance studies showed that F1 plants of $3707 / 5 \mathrm{XPh}-0$ are partially resistant to late blight (show a disease severity rate lower than $\mathrm{Ph}-0$ but higher than 3707/5) and F2 plants segregate in a way that supports the hypothesis of resistance in L3707/5 being controlled by two independent genes: a partially dominant gene and a dominant, epistatic gene (16).

The objective of the present study was to elucidate whether recombinant isolates of $P$. infestans exhibit altered pathogenic fitness to tomato genotypes carrying various genes for late blight resistance. More specifically, we wanted to reveal whether or not S. pimpinellifolium L3707/5 is resistant to oosporic progeny isolates of $P$. infestans, presuming that sexual reproduction of this pathogen may produce recombinant isolates with enhanced pathogenic fitness. Such information could be very important to tomato breeders who use this material in their late-blight-resistance breeding programs. To achieve this, oospores were produced in vivo and the pathogenic fitness of progeny isolates and their parents was tested on four tomato genotypes carrying different major genes for resistance: $P h-O$ (no $R$ genes), $P h-1$, $P h-2$, or 3707.

Crosses and progeny isolates. Oospores were produced in detached tomato (S. esculentum L.) leaves by inoculation with a mixture (1:1) of A1 and A2 sporangia, as described previously (8). Four parental field isolates, all A1, sensitive to

\section{MATERIALS AND METHODS}

metalaxyl and mefenoxam and identified as $\mathrm{AD}, \mathrm{SU}, \mathrm{T} 04$, and 96B were each crossed with an A2 field isolate identified as Pi367 (formerly 367) resistant to metalaxyl and mefenoxam. The origin of these isolates is given elsewhere (29). Oospores were extracted, exposed to two cycles of drying and wetting as described previously (29), and used to infect tomato leaves floating on perlite-oospore cultures. Twenty-nine lesions (referred to from here on as "families") were selected from the four crosses and then 283 singlesporangium isolates (SSIs) were obtained and analyzed (Table 1). Parental isolates and SSIs were maintained by repeated inoculation onto detached tomato leaves (cv. HA-870) in petri dishes incubated at $14^{\circ} \mathrm{C}$ with $12 \mathrm{~h}$ of light/day and kept moist by adding $1 \mathrm{ml}$ of water/dish every 3 days. Each isolate was tested for resistance to metalaxyl and mefenoxam, mating type, and pathogenic fitness on tomato. Information on each isolate in regard to mating type and sensitivity or resistance to metalaxyl and mefenoxam has been published previously (29). Most progeny isolates exhibited intermediate resistance to metalaxyl and most belonged to either the A1 or A2 mating type, which confirmed their oosporic, recombinant origin (29).

Pathogenic fitness of progeny isolates. Pathogenic fitness was estimated in leaf discs of tomato according to the method described by Kadish and Cohen for potato, with modifications (17). The leaf disc method has been used successfully $(17,19)$ to estimate pathogenic fitness of metalaxyl-sensitive and metalaxyl-resistant isolates of $P$. infestans from potato, to monitor the proportion of metalaxylresistant sporangia in a mixed sporangial suspension of metalaxyl-sensitive and metalaxyl-resistant isolates, and to monitor

Table 1. Crosses made between field isolates Pi367 (A2 mating type, metalaxyl-resistant) and AD, SU, T04, and 96 B (all A1 mating type, metalaxyl-sensitive) of Phytophthora infestans ${ }^{\mathrm{a}}$

\begin{tabular}{|c|c|c|c|c|c|c|c|}
\hline \multicolumn{8}{|c|}{ Cross } \\
\hline \multicolumn{2}{|c|}{$\mathrm{Pi367} \times \mathrm{AD}$} & \multicolumn{2}{|c|}{$\mathrm{Pi} 367 \times \mathrm{SU}$} & \multicolumn{2}{|c|}{$\mathrm{Pi367} \times \mathrm{T04}$} & \multicolumn{2}{|c|}{$\mathrm{Pi} 367 \times 96 \mathrm{~B}$} \\
\hline Code & SSIs & Code & SSIs & Code & SSIs & Code & SSIs \\
\hline 267 & 15 & 277 & 13 & S4 & 20 & 283 & 10 \\
\hline 268 & 17 & 278 & 11 & S5 & 20 & $\mathrm{~S} 12$ & 6 \\
\hline 269 & 10 & 279 & 7 & S11 & 10 & $\ldots$ & $\ldots$ \\
\hline 270 & 4 & 280 & 12 & $\ldots$ & $\ldots$ & $\ldots$ & $\ldots$ \\
\hline 271 & 3 & 281 & 4 & $\ldots$ & $\ldots$ & $\ldots$ & $\ldots$ \\
\hline 273 & 8 & 282 & 8 & $\ldots$ & $\ldots$ & $\ldots$ & $\ldots$ \\
\hline 275 & 15 & S0 & 13 & $\ldots$ & $\ldots$ & $\ldots$ & $\ldots$ \\
\hline 276 & 4 & S2 & 6 & $\ldots$ & $\ldots$ & $\ldots$ & $\ldots$ \\
\hline $276 \mathrm{~A}$ & 11 & S3 & 5 & $\ldots$ & $\ldots$ & $\ldots$ & $\ldots$ \\
\hline S1 & 18 & S8 & 3 & $\ldots$ & $\ldots$ & $\ldots$ & $\ldots$ \\
\hline S6 & 5 & S9 & 11 & $\ldots$ & $\ldots$ & $\ldots$ & $\ldots$ \\
\hline S7 & 9 & $\ldots$ & $\ldots$ & $\ldots$ & $\ldots$ & $\ldots$ & $\ldots$ \\
\hline S10 & 5 & & $\ldots$ & & $\ldots$ & . & $\ldots$ \\
\hline $\mathrm{T}=13$ & $\mathrm{~T}=124$ & $\mathrm{~T}=11$ & $\mathrm{~T}=93$ & $\mathrm{~T}=3$ & $\mathrm{~T}=50$ & $\mathrm{~T}=2$ & $\mathrm{~T}=16$ \\
\hline
\end{tabular}

a Numbers represent the family codes for the progeny families obtained via perlite-tomato leaf bait cultures (each family originated from a single lesion) and the number of single-sporangium isolates (SSIs) produced from each family that were used in the study. $\mathrm{T}=$ total number of families and SSIs per cross. sensitivity of $P$. infestans progeny isolates to metalaxyl.

Four tomato genotypes, susceptible and resistant to $P$. infestans, were used: Hazera 870 (Hazera Genetics, Mivhor, Israel) without resistance to late blight, $P h-0$; New Yorker, carrying $P h-1$; Pierline, carrying $P h-2$ (provided by M. Pitrat, INRA, Monfavet, France); and S. pimpinellifolium L3707/5, which carries two independent genes for resistance (they provide partial resistance when heterozygote) (16). Tomato plants were grown in the greenhouse to the 12-leaf stage. Leaves six to nine from the stem base were detached and leaf discs (22 $\mathrm{mm}$ in diameter) were removed with the aid of a cork borer and placed on wet filter paper in 9-cm petri dishes (five per dish) with the lower surface uppermost. Each was then inoculated with one 10- $\mu \mathrm{l}$ droplet (containing 20 sporangia) of the appropriate sporangial suspension. Dishes were incubated at $18^{\circ} \mathrm{C}(12 \mathrm{~h}$ of light/day). At 7 days post inoculation (dpi), each disc was placed in $2 \mathrm{ml}$ of $50 \%$ ethanol and agitated for $10 \mathrm{~min}$, and the dislodged sporangia were counted with the aid of a hemacytometer.

Statistical analysis. Three independent experiments were conducted. After testing the homogeneity of variance among the independent experiments, the data were combined for further analysis. Mean number of sporangia per square centimeter of leaf tissue ( \pm standard deviation $[S D]$ ) for each SSI on each tomato line was obtained. These means were also used to calculate the mean number of sporangia per square centimeter $( \pm \mathrm{SD})$ for all SSIs derived from each particular lesion, (3 to 20 SSIs per family, 2 to 13 families per cross, total 29 families; Table 1) on each tomato line (Fig. 1). The family-genotype interaction effect on the sporulation was estimated using the general linear model (GLM) procedure of the SAS package. The linear model used for analysis comprised the components family, genotype, family-genotype interaction, and residual. Means were subjected to Waller-Duncan K-ratio $t$ test $(\alpha=0.05)$ to establish the significant difference among the four tomato genotypes. Sporangial counts of family 267, derived from the cross $\mathrm{Pi} 367 \times$ $\mathrm{AD}$, were subjected to one-way analysis of variance (ANOVA) and means were separated with Fisher's protected least significant difference analysis $(\alpha=0.05)$ to establish significant differences between SSIs on each tomato line (Fig. 2). The fitness of the other 28 families (268 isolates) is presented in a concise way: minimum, maximum, and median fitness values for all isolates in each family, relative to their parents, on each tomato line (Figs. 3-5).

\section{RESULTS}

Data in Figure 1 represent the mean sporangial production of all SSIs in a family 
(total of 29 families) on each tomato genotype. The number of SSIs per family is given in Table 1 . The grand mean sporulation values (mean of 29 means) of all SSIs were significantly different $(P<0.0001)$ on Ph-0, Ph-1, Ph-2, and 3707: $35.0 \pm 14.8$, $21.2 \pm 9.3,4.1 \pm 2.9$ and $0.6 \pm 1.7 \times 10^{3}$ sporangia $/ \mathrm{cm}^{2}$, respectively. The familygenotype interaction and the family effect on sporulation were not significant $(\mathrm{F}=$ $0.67, P=0.737$ and $\mathrm{F}=0.75, P=0.528$, respectively)

Isolate $\mathrm{Pi} 367$, the $\mathrm{A} 2$ parent of all crosses, produced a mean of $62.5 \pm 5.0 \times$ $10^{3}$ sporangia/ $\mathrm{cm}^{2}$ on $P h-O$ while the A1 parents $\mathrm{AD}, \mathrm{SU}, \mathrm{T} 04$, and $96 \mathrm{~B}$ produced significantly smaller $(\alpha=0.05)$ sporangial yields: $27 \pm 2,26 \pm 3,22.5 \pm 4$ and $22.8 \pm$ $6 \times 10^{3}$ sporangia/ $/ \mathrm{cm}^{2}$, respectively, on the same cultivar (Fig. 1). All parental isolates were capable of sporulating on $P h-l$ but intensity of sporulation declined relative to $P h-0$ by $33,56,12,19$, and $30 \%$ for Pi367, AD, SU, T04, and 96B, respectively (Fig. 1). The A2 parent Pi367 produced a significantly larger $(\alpha=0.05)$ yield of sporangia on $P h-1$ compared with all A1 isolates, except SU. Sporulation of the parental isolates declined further on $P h-2$
(Fig. 1), with no significant differences between isolates, except isolate T04, which produced no sporangia on $P h-2$. Sporulation of parental isolates was totally diminished on 3707 (Fig. 1) but minute necrotic spots (hypersensitive resistance) were seen on the leaf discs beneath the inoculum droplets.

The results obtained with 15 progeny isolates of one such lesion (family), 267, derived from the cross $\mathrm{Pi} 367 \times \mathrm{AD}$, are illustrated in more detail in Figure 2. The pathogenic fitness of these SSIs (arranged in a descending order) differed significantly from one another and were also significantly different on the four tomato lines. Sporulation was 45 to 100,14 to 64 , 3 to 10 , and 0 to 31 sporangia/ $/ \mathrm{cm}^{2}$ on $P h-O$, $P h-1, P h-2$, and 3707 , respectively, with a mean $( \pm \mathrm{SD})$ of $70.5 \pm 19.2,34.9 \pm 12.9$, $7.0 \pm 2.3$, and $8.8 \pm 9.6$ sporangia $/ \mathrm{cm}^{2}$, respectively. ANOVA indicated significant differences $(\alpha=0.05)$ between $P h-0, P h-1$, and $P h-2$ but not between $P h-2$ and 3707 . The data also identified progeny isolates with pathogenic fitness higher or lower than the parents $\mathrm{Pi} 367$ and $\mathrm{AD}$, respectively. On $P h-0,11$ isolates exhibited a higher fitness than Pi367 (the fitter A2 parent) whereas, on $P h-2,12$ isolates suffered reduced fitness compared with $\mathrm{AD}$ (the less-fit A1 parent). Most importantly, 12 of 15 isolates were capable of sporulating on 3707 , on which neither parent could sporulate.

The $\mathrm{x}$ axes in Figure 2A, B, C, and D also show that the order of the isolates in each graph is different. This means that the pathogenic fitness of an isolate on one tomato line did not necessarily correlate with the pathogenic fitness on another tomato line. For example, SSI 4 showed the highest fitness on $P h-O$, moderate fitness on $P h-2$, and the lowest fitness on $P h$ 1 and 3707. SSI 8, which was most fit on 3707 , was moderately fit on the other tomato genotypes.

Cross Pi367 $\times$ AD. Thirteen lesions (families) were obtained from three crosses of Pi367 $\times \mathrm{AD}$, from which 124 SSIs of $P$. infestans were produced and tested (Table 1). The pathogenic fitness of these isolates on tomato lines $P h-0, P h-l$, $P h-2$, or 3707 is presented in Figures 1 and 3 . The A2 parent Pi367 produced significantly more $(\alpha=0.05)$ sporangia per unit leaf area than the A1 parent AD on $P h-O$ and $P h-1$ but an equally low number of
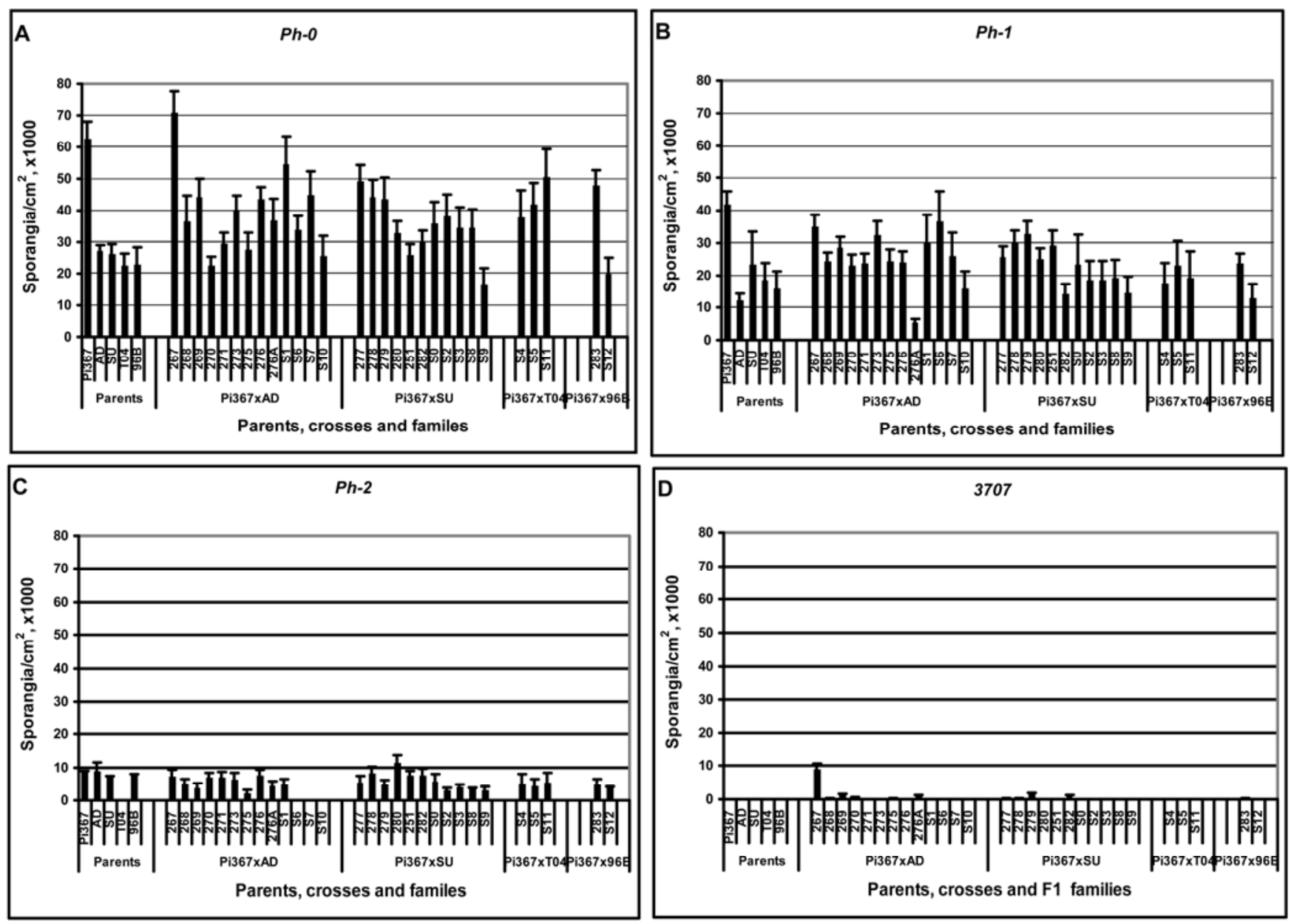

Fig. 1. Mean pathogenic fitness values of five parental isolates and 29 progeny families (total of 283 single-sporangium isolates) of Phytophthora infestans derived from four crosses on four tomato lines ( $P h-0, P h-1, P h-2$, and 3707) carrying different genes for resistance to late blight. Values represent mean pathogenic fitness (sporangia/ $\mathrm{cm}^{2}$ and standard deviation of the mean) of all isolates in each family of each cross on each tomato line as measured in three independent experiments. 

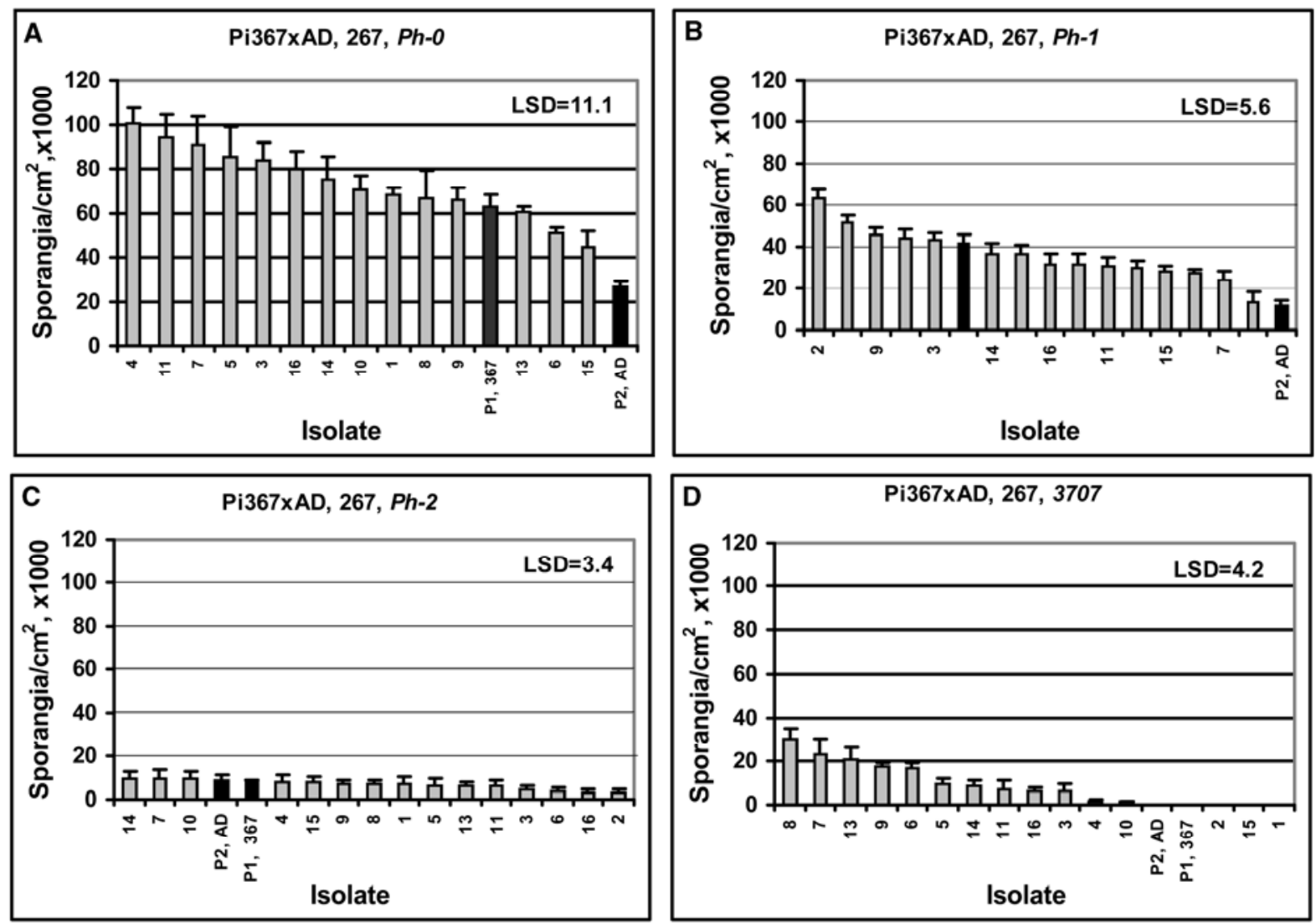

Fig. 2. Pathogenic fitness (sporangia/ $\mathrm{cm}^{2}$ ) of 15 single-sporangium progeny isolates (SSIs) of Phytophthora infestans derived from family 267 of the cross $\mathrm{Pi} 367 \times \mathrm{AD}$ on four tomato lines carrying different genes for resistance to late blight. Black columns represent the parent isolates Pi367 and AD. Columns and bars on columns represent mean and the standard deviation of the mean for each isolate as measured in three independent experiments. Least significant difference (LSD) values on the upper right side of each panel represent the LSD between SSIs at $\alpha=0.05$. Mean values of pathogenic fitness for all 15 SSIs on $P h-0, P h-1, P h-2$, and 3707 tomato is $74.2 \pm 16.2,35.9 \pm 11.0,6.8 \pm 1.7$, and $10.0 \pm 9.5 \times 10^{3}$ sporangia/ $\mathrm{cm}^{2}$, respectively (see also Figure 1). Analysis of variance assigned the letters $\mathrm{A}, \mathrm{B}, \mathrm{C}$, and $\mathrm{C}$ to these means (not shown).
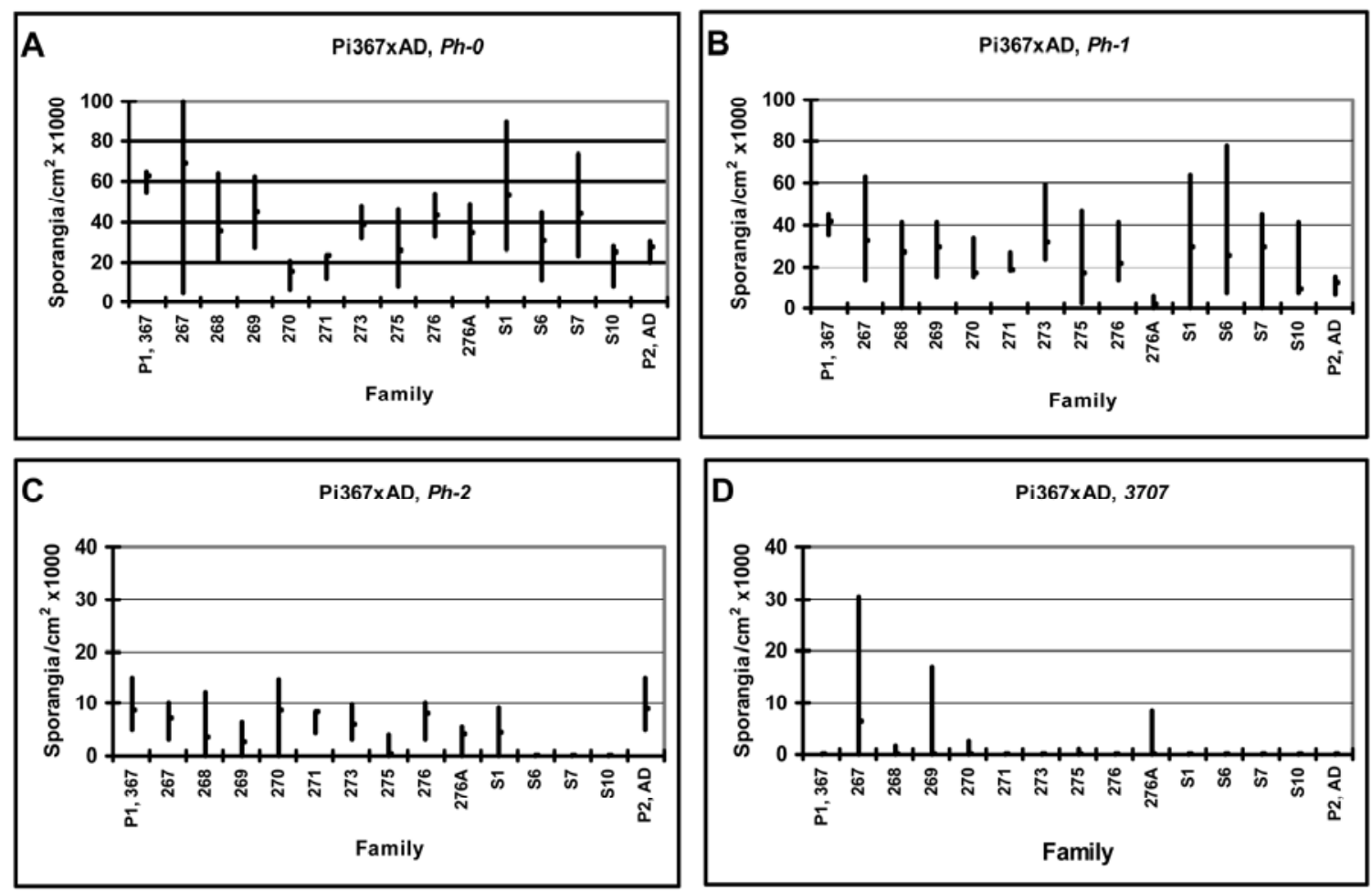

Fig. 3. Mean minimum, mean maximum, and median values of pathogenic fitness (sporangia/ $\mathrm{cm}^{2} \times 10^{3}$ ) of parental isolates Pi367 and AD of Phytophthora infestans and 13 progeny lesions (families) on four tomato lines (Ph-0,Ph-1,Ph-2, and 3707) with different genes for resistance to late blight. For number of isolates in a family, see Table 1 . Note the different scales on the y axis. 
sporangia on $P h-2$. Both parents failed to sporulate on 3707 . The vertical lines in Figure 3 indicate the lowest (minimum) and the highest (maximum) fitness values measured among the isolates derived from each lesion (family). The dot on each line indicates the median fitness value. The number of families that developed on $P h-0, P h-1, P h-2$, and 3707 was $13,13,10$, and 6 , respectively. Of the six families that developed on 3707 (on which the parents Pi367 and AD did not), three $(267,269$, and 276A) produced more than $5 \times 10^{3}$ sporangia $/ \mathrm{cm}^{2}$. The mean pathogenic fitness values of all 13
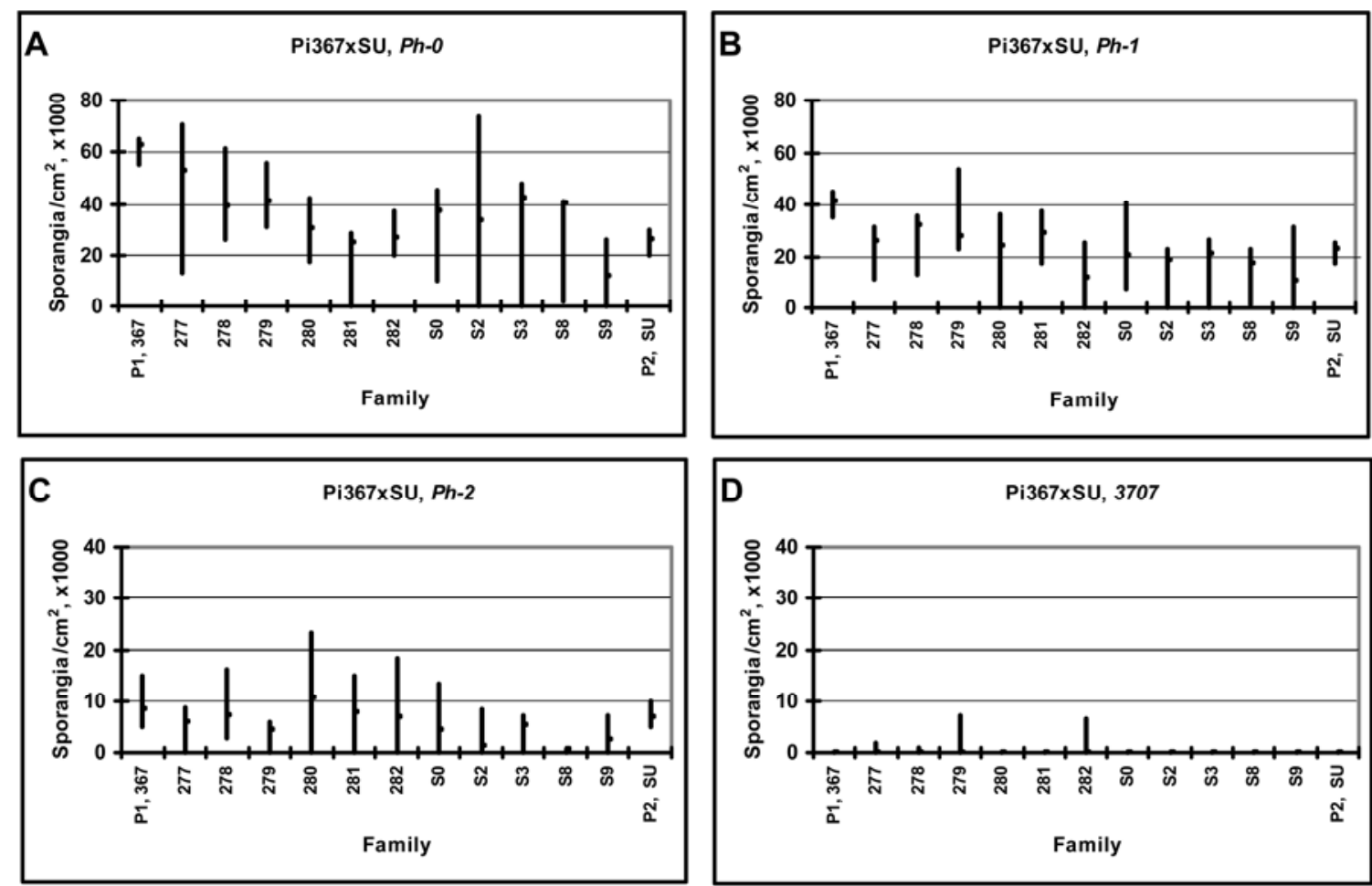

Fig. 4. Mean minimum, mean maximum, and median values of pathogenic fitness (sporangia/ $\mathrm{cm}^{2} \times 10^{3}$ ) of parental isolates Pi367 and SU of Phytophthora infestans and 11 lesions (families) on four tomato lines ( $P h-0, P h-1, P h-2$, and 3707) with different genes for resistance to late blight. For number of isolates in a family, see Table 1 . Note the different scales on the y axis.
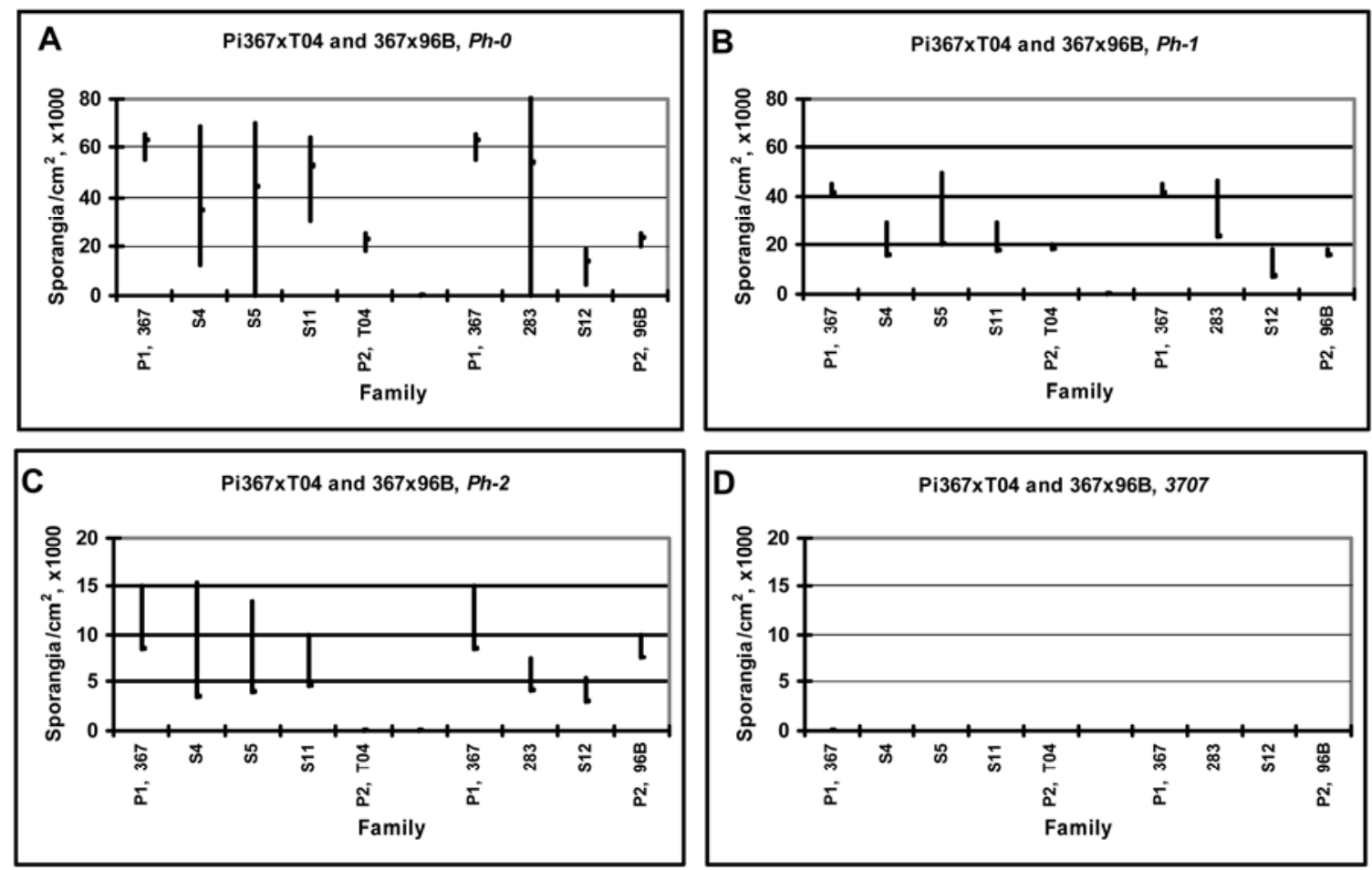

Fig. 5. Mean minimum, mean maximum, and median values of pathogenic fitness (sporangia/ $\mathrm{cm}^{2} \times 10^{3}$ ) of parental isolates Pi367 and T04 plus Pi367 and $96 \mathrm{~B}$ of Phytophthora infestans and three and two lesions (families), respectively, on four tomato lines $(P h-0, P h-1, P h-2$, and 3707$)$ with different genes for resistance to late blight. For number of isolates in a family, see Table 1. Note the different scales on the y axis. 
families decreased in a significant manner $(\alpha=0.05)$ in the order $P h-O>P h-1>P h$ $2>3707$ (Fig. 1).

Cross Pi367 $\times$ SU. Eleven lesions were obtained from six crosses of Pi367 $\times$ SU, from which 93 SSIs of $P$. infestans were produced and tested (Table 1). The pathogenic fitness of these isolates on tomato lines $P h-0, P h-1, P h-2$, or 3707 is presented in Figures 1 and 4 . The vertical lines in Figure 4 show that 11, 11, 11, and 4 families developed on $P h-O, P h-1, P h-2$, and 3707, respectively. Mean pathogenic fitness values of all 11 families decreased in a significant manner $(\alpha=0.05)$ in the order $P h-O>P h-1>P h-2>3707$ (Fig. 1).

Cross Pi367 $\times$ T04. Three lesions were obtained from two crosses of Pi367 $\times$ T04, from which 50 SSIs of $P$. infestans were produced and tested (Table 1). Pathogenic fitness of these isolates (three families) on $P h-0, P h-1, P h-2$, or 3707 is presented in Figures 1 and 5. The A1 parent T04 failed to survive on either $P h-2$ or 3707 . In all, 3 , 3, 3, and 0 families developed on $P h-0$, $P h-1, P h-2$, and 3707, respectively (Fig. 5). Mean pathogenic fitness values of all three families decreased in a significant manner $(\alpha=0.05)$ in the order Ph-O $>P h$ $1>P h-2>3707$ (Fig. 1).

Cross Pi367 $\times$ 96B. Two lesions were obtained from two crosses of Pi367 $\times 96 \mathrm{~B}$, from which 16 SSIs of $P$. infestans were produced and tested (Table 1). Pathogenic fitness of these families on $P h-0, P h-1, P h$ 2, or 3707 is presented in Figures 1 and 5. Mean pathogenic fitness values of the two families decreased in a significant manner $(\alpha=0.05)$ in the order $P h-O>P h-1>P h-2$ $>3707$ (Fig. 1).

Fitness of progeny and parental isolates. Table 2 classifies the 283 progeny isolates into three categories: isolates with pathogenic fitness higher than the A2 parent, those with pathogenic fitness lower than the A1 parent, and those with pathogenic fitness between the A2 and A1 parents. The data were extracted from the mean pathogenic fitness records of each SSI (not shown). They indicate that 25.6, 43.1 , and $57.6 \%$ of all progeny isolates exhibited a reduced pathogenic fitness relative to their A1 parent, whereas 11.7, 6.4 , and $14.5 \%$ attained a higher pathogenic fitness relative to the A2 parent on
$P h-0, P h-1$, and $P h-2$, respectively. Thirtyseven isolates $(13.1 \%$ of all progeny isolates) gained pathogenic fitness on 3707 , on which no parent was fit. Overall, reduced fitness was more frequent among the progeny isolates than increased fitness.

\section{DISCUSSION}

The mode of inheritance of mating type, haplotype, enzyme isoforms, aggressiveness and resistance to metalaxyl have been reported for in vitro-produced F1 progeny isolates of $P$. infestans $(2,15,19,21,30,36)$. However, the pathogenic fitness of in vivoproduced $\mathrm{F} 1$ progeny isolates to potato or tomato have not been studied in spite of the documented genotypic differences between in vivo- and in vitro-produced oospores of $P$. infestans (36). Such studies may provide a better understanding of the current or future composition of $P$. infestans populations. In the United States, isolates of $P$. infestans with higher fitness, that had probably migrated from Mexico (12), were shown to replace isolates with lower fitness, causing major shifts in pathogen populations. Almost all isolates of this pathogen collected from the $\mathrm{Co}-$ lumbia Basin, Idaho, in 1992 belonged to the US-1 genotype, whereas $97 \%$ were identified as US-8 by 1995 (22). Miller et al. (23) compared the aggressiveness level of 22 isolates from different clonal lineages, including six US-1 isolates and three US- 8 isolates, on four potato cultivars with different levels of quantitative resistance. They found that US-8 isolates had a higher lesion expansion rate, a higher sporulation capacity and a shorter latent period than US-1 isolates.

In this article, we report on the pathogenic fitness of progeny isolates of $P$. infestans to tomato lines carrying major genes for late blight resistance. Twentynine lesions of late blight were derived from oospore infection of tomato leaves; in total, 283 SSIs were derived from these lesions and tested for pathogenic fitness. The results showed a large variation in sporangial production per unit leaf area (our measure for pathogenic fitness) on the four genotypes of tomato; many progeny isolates lost pathogenic fitness relative to their A1 parent whereas others acquired enhanced pathogenic fitness relative to their A2 parent. Most importantly, 37 progeny isolates $(13.1 \%)$ derived from 11 lesions of three (out of four) crosses were capable of sporulating on the multiple-race resistant tomato line 3707. No parental isolate of $P$. infestans could sporulate on this line, nor was this line susceptible under natural conditions. This result suggests that the resistance of this line to late blight might not be durable in regions where $P$. infestans reproduces sexually. This report further demonstrates that oosporic progeny isolates produced under controlled conditions in vivo can acquire pathogenicity to a resistant tomato line. Similarly, a "breakdown" of resistance also occurred in the multiple-race resistant potato carrying an $R$ gene ( $R B$ or Rpi-blb1) from $S$. bulbocastanum (12).

Based on our previous data (29), we assume that the 283 isolates studied here are true F1 hybrid isolates. They were intermediately resistant to metalaxyl while their parents were either sensitive or resistant to this fungicide. The variation in sporulation intensity among progeny isolates derived from the same lesion (family) likely resulted from true genetic sexual recombination rather than somatic recombination, where different sporangial isolates are propagated from the same oospore lesion.

Large variation among F1 progeny isolates was also reported for lesion size (15) and resistance to metalaxyl (19). Caten and Jinks (3) reported on variation in growth rate and sporangium production on artificial medium among single zoospore cultures of $P$. infestans in contrast to single sporangia or single hyphal tips, which were much more uniform, and suggested "cytoplasmic changes" as the reason for variation, Niederhauser (25) reported on segregation for mating type and virulence factors among single-zoospore cultures of $P$. infestans.

The fact that cv. New Yorker carrying $P h-1$ and cv. Pierline carrying $P h-2$ allowed for reduced sporulation relative to $P h-0$, although both are susceptible to late blight under Israeli conditions (6), supports the idea cited by Tan (34) that defeated $R$ genes may express residual resistance and still be valuable in breeding programs.

Our previous study (16) showed that the inheritance of resistance against $P$. in-

Table 2. Number and percentage of single-sporangium isolates (SSIs) in each of four crosses showing higher, intermediate, or lower pathogenic fitness than their respective parents P1 (Pi367, A2 mating type, resistant to metalaxyl and mefenoxam) and P2 (AD, SU, T04, or 96B, all A1 mating type, sensitive to metalaxyl and mefenoxam) as measured on four tomato genotypes with late blight resistance genes $P h-0, P h-1, P h-2$, and $3707^{\mathrm{a}}$

\begin{tabular}{|c|c|c|c|c|c|c|c|c|c|c|c|c|c|}
\hline \multirow[b]{2}{*}{ Cross } & \multirow[b]{2}{*}{ SSIs $^{\mathbf{b}}$} & \multicolumn{3}{|c|}{$P h-0$} & \multicolumn{3}{|c|}{$P h-1$} & \multicolumn{3}{|c|}{$P h-2$} & \multicolumn{3}{|c|}{3707} \\
\hline & & $>\mathbf{P 1}$ & $<$ P1 $>$ P2 & $<\mathbf{P 2}$ & $>\mathbf{P 1}$ & $<\mathrm{P} 1>\mathrm{P} 2$ & $<\mathbf{P 2}$ & $>\mathbf{P 1}$ & $<$ P1 $>$ P2 & $<\mathbf{P 2}$ & $>\mathbf{P 1}$ & $<\mathbf{P} 1>\mathbf{P} 2$ & $<\mathbf{P 2}$ \\
\hline $\mathrm{Pi} 367 \times \mathrm{AD}$ & 124 & 20 & 70 & 31 & 14 & 74 & 36 & 10 & 0 & 94 & 24 & 0 & 0 \\
\hline $\mathrm{Pi} 367 \times \mathrm{SU}$ & 93 & 3 & 62 & 28 & 2 & 43 & 48 & 22 & 17 & 54 & 11 & 0 & 0 \\
\hline Pi367 × T04 & 50 & 7 & 36 & 7 & 1 & 21 & 28 & 8 & 42 & 0 & 0 & 0 & 0 \\
\hline Pi367 × 96B & 16 & 3 & 7 & 7 & 1 & 5 & 10 & 1 & 0 & 15 & 2 & 0 & 0 \\
\hline Total & 283 & 33 & 175 & 73 & 18 & 143 & 122 & 41 & 59 & 163 & 37 & 0 & 0 \\
\hline Percent & $\ldots$ & 11.7 & 61.8 & 25.8 & 6.4 & 50.5 & 43.1 & 14.5 & 20.8 & 57.6 & 13.1 & 0.0 & 0.0 \\
\hline
\end{tabular}

a Pathogenic fitness was measured by counting the number of sporangia per square centimeter produced on tomato leaf discs in three independent experiments.

b Total number of SSIs. 
festans in S. pimpinellifolium L3707/5 is controlled by two genes, a semidominant $R$ gene and a dominant epistatic $(E)$ gene required for the function of $R$. Based on this model, one recombination event in $P$. infestans may overcome the resistance of 3707. Heterozygote parents that carry one avirulence allele ( $A v r$, dominant, corresponding to either $R$ or $E$ ) in a locus will produce, upon mating, progeny isolates compatible with 3707.

In Israel, severe epidemics of late blight have occurred in tomato since 1999. Serious epidemics were also reported in Europe (20), California (14), and Taiwan (4). Isolates of $P$. infestans collected from tomato in Israel during 1997 to 2000 (6) were mostly complex races (up to eight virulence factors), highly aggressive to tomato lines with $P h-O$ and $P h-1$ and moderately aggressive to lines with $P h-2$. Isolates collected in 2007 carried, for the first time, up to 10 virulence factors (9). Smart and Fry (31) concluded that "the increased pathogenic fitness of $P$. infestans strains has emphasized the need to develop resistant plants as an important component in the management of this disease." Chen et al. (4) concluded that "breeding new tomato cultivars for resistance to $P$. infestans is an urgent and ongoing need because new races of the pathogen appeared continuously...." Unfortunately, as shown here, resistant genotypes of tomato may not necessarily stop the development of new recombinant isolates of $P$. infestans, and this emphasizes the need to incorporate diverse sources of resistance into breeding programs, including defeated $R$ genes which may contribute residual resistance (34). This need is especially true in areas negatively affected by sexually reproducing populations of $P$. infestans.

In conclusion, the present study confirmed that controlled crosses made in vivo between A1 and A2 isolates of P. infestans produced progeny isolates with high pathogenic fitness to otherwise resistant tomato cultivars. Such crosses also produced many isolates with reduced pathogenic fitness to tomato. The fate of such isolates in nature and their fitness to potato or tomato is not yet known.

\section{LITERATURE CITED}

1. Black, L. L., Wang, T. C., and Huang, Y. H. 1996. New sources of late blight resistance identified in wild tomatoes. Trop. Veg. Inf. Serv. Newsl. 1:15-17.

2. Carter, D. A., Buck, K. W., Archer, S. A., Van der Lee, T., Shattock, R. C., and Shaw, D. S. 1999. The detection of nonhybrid, trisomic, and triploid offspring in sexual progeny of a mating of Phytophthora infestans. Fungal Genet. Biol. 26:198-208.

3. Caten, C. E., and Jinks, J. L. 1968. Spontaneous variability of single isolates of Phytophthora infestans. I. Cultural variation. Can. J. Bot. 46:329-348.

4. Chen, C. H., Sheu, Z. M., and Wang, T. C. 2008. Host specificity and tomato-related race composition of Phytophthora infestans isolates in Taiwan during 2004 and 2005. Plant Dis. 92:751-755.

5. Chunwongse, J., Chunwongse, C., Black, L., and Hanson, P. 2002. Molecular mapping of the $P h-3$ gene for late blight resistance in tomato. J. Hortic. Sci. Biotechnol. 77:281-286.

6. Cohen, Y. 2002. Populations of Phytophthora infestans in Israel underwent three major genetic changes during 1983 to 2000. Phytopathology 92:300-307.

7. Cohen, Y., Farkash, S., Baider, A., and Shaw, D. S. 2000. Sprinkling irrigation enhances production of oospores of Phytophthora infestans in field-grown crops of potato. Phytopathology 90:1105-1111.

8. Cohen, Y., Farkash, S., Reshit, Z., and Baider, A. 1997. Oospore production of Phytophthora infestans in potato and tomato leaves. Phytopathology 87:191-196.

9. Cohen, Y., Galperin, M., and Rubin, A. E. 2009. RAPD, mating type, virulence and resistance to metalaxyl in Phytophthora infestans in Israel. (Abstr.) Phytoparasitica 37.

10. Drenth, A., Janssen, E. M., and Govers, F. 1995. Formation and survival of oospores of Phytophthora infestans under natural conditions. Plant Pathol. 44:86-94.

11. Fernandez-Pavia, S. P., Grunwald, N. J., DiazValasis, M., Cadena-Hinojosa, M., and Fry, W. E. 2004. Soilborne oospores of Phytophthora infestans in central Mexico survive winter fallow and infect potato plants in the field. Plant Dis. 88:29-33.

12. Fry, W. E. 2008. Phytophthora infestans: the plant (and $R$ gene) destroyer. Mol. Plant Pathol. 9:385-402.

13. Gallegly, M. E., and Galindo, J. 1958. Mating types and oospores of Phytophthora infestans in nature in Mexico. Phytopathology 48:274277.

14. Gavino, P. D., Smart, C. D., Sandrock, R. W., Miller, J. S., Hamm, P. B., Lee, T. Y., Davis, R. M., and Fry, W. E. 2000. Implications of sexual reproduction for Phytophthora infestans in the United States: generation of an aggressive lineage. Plant Dis. 84:731-735.

15. Gisi, U., and Cohen, Y. 1996. Resistance to phenylamide fungicides: a case study with Phytophthora infestans involving mating type and race structure. Annu. Rev. Phytopathol. 34:549-572.

16. Irzhansky, I., and Cohen, Y. 2006. Inheritance of resistance against Phytophthora infestans in Lycopersicon pimpenellifolium L3707. Euphytica 149:309-316.

17. Kadish, D., and Cohen, Y. 1988. Estimation of metalaxyl-resistance in Phytophthora infestans. Phytopathology 78:915-919.

18. Kim, M. J., and Mutschler, M. A. 2006. Characterization of late blight resistance derived from Solanum pimpinellifolium L3708 against multiple isolates of the pathogen Phytophthora infestans. J. Am. Soc. Hortic. Sci. 131:637645.

19. Knapova, G., Schlenzig, A., and Gisi, U. 2002. Crosses between isolates of Phytophthora infestans from potato and tomato and characterization of F-1 and F-2 progeny for phenotypic and molecular markers. Plant Pathol. 51:698709.

20. Legard, D. E., Lee, T. Y., and Fry, W. E. 1995. Pathogenic specialization in Phytophthora infestans: aggressiveness on tomato. Phytopathology 85:1356-1361.

21. Mayton, H., Smart, C. D., Moravec, B. C., Mizubuti, E. S. G., Muldoon, A. E., and Fry, W. E. 2000. Oospore survival and pathogenicity of single oospore recombinant progeny from a cross involving US-17 and US-8 genotypes of Phytophthora infestans. Plant Dis.
84:1190-1196.

22. Miller, J. S., Hamm, P. B., and Johnson, D. A. 1997. Characterization of the Phytophthora infestans population in the Columbia Basin of Oregon and Washington from 1992 to 1995. Phytopathology 87:656-660.

23. Miller, J. S., Johnson, D. A., and Hamm, P. B. 1998. Aggressiveness of isolates of Phytophthora infestans from the Columbia Basin of Washington and Oregon. Phytopathology 88:190-197.

24. Moreau, P., Thoquet, P., Olivier, J., Laterrot, H., and Grimsley, N. 1998. Genetic mapping of $P h-2$, a single locus controlling partial resistance to Phytophthora infestans in tomato. Mol. Plant-Microbe Interact. 11:259-269.

25. Niederhauser, J. S., Lucas, J. A., Shattock, R. C., Shaw, D. S., and Cooke, L. R. 1991. Phytophthora infestans: The Mexican connection. Pages 25-45 in: Phytophthora. British Mycological Society, Cambridge University Press, Cambridge.

26. Rubin, E., Baider, A., and Cohen, Y. 2001. Phytophthora infestans produces oospores in fruits and seeds of tomato. Phytopathology 91:1074-1080.

27. Rubin, E., and Cohen, Y. 2004. Oospores associated with tomato seed may lead to seedborne transmission of Phytophthora infestans. Phytoparasitica 32:237-245.

28. Rubin, E., and Cohen, Y. 2004. Phenotypic characterization of oosporic isolates of Phy tophthora infestans derived from tomato leaves. Phytoparasitica 32:190-191.

29. Rubin, E., and Cohen, Y. 2006. An improved method for infecting tomato leaves or seedlings with oospores of Phytophthora infestans used to investigate F1 progeny. Plant Dis. 90:741-749.

30. Shattock, R. C. 2002. Phytophthora infestans: populations, pathogenicity and phenylamides. Pest Manage. Sci. 58:944-950.

31. Smart, C. D., and Fry, W. E. 2004. Invasions by the late blight pathogen: renewed sex and enhanced fitness. Biol. Invasions 3:235-243.

32. Stromberg, A., Peterson, L., and Wikstrom, M 1999. Infection of potato by oospores of Phytophthora infestans in soil. Plant Dis. 83:876.

33. Sujkowski, L. S., Goodwin, S. B., Dyer, A. T., and Fry, W. E. 1994. Increased genotypic diversity via migration and possible occurrence of sexual reproduction of Phytophthora infestans in Poland. Phytopathology 84:201-207.

34. Tan, M. Y. A., Hutten, R. C. B., Celis, C., Park, T. H., Niks, R. E., Visser, R. G. F., and van Eck, H. J. 2008. The RPi-mcd1 locus from Solanum microdontum involved in resistance to Phytophthora infestans, causing a delay in infection, maps on potato chromosome 4 in a cluster of NBS-LRR genes. Mol. PlantMicrobe Interact. 21:909-918.

35. Turkensteen, L. J., Flier, W. G., Wanningen, R., and Mulder, A. 2000. Production, survival and infectivity of oospores of Phytophthora infestans. Plant Pathol. 49:688-696.

36. Van der Lee, T., Testa, A., Robold, A., 't Klooster, J., and Govers, F. 2004. High-density genetic linkage maps of Phytophthora infestans reveal trisomic progeny and chromosomal rearrangements. Genetics 167:16431661

37. Zarzycka, H., and Sobkowiak, S. 1997. Formation and survival of Phytophthora infestans oospores and their role as a primary infection source of the pathogen. Plant Breed. Seed Sci. 41:27-38.

38. Zwankhuizen, M. J., Govers, F., and Zadoks, J. C. 2000. Inoculum sources and genotypic diversity of Phytophthora infestans in Southern Flevoland, the Netherlands. Eur. J. Plant Pathol. 106:667-680. 